\title{
Short-term effects of basolateral glucosensing on sodium-dependent glucose uptake in ruminal epithelia of sheep*
}

\author{
J.R. Aschenbach ${ }^{1,3}$, C. Atasoglu ${ }^{2}$, T. Borau ${ }^{1}$ and G. Gäbel ${ }^{1}$ \\ ${ }^{1}$ Institute of Veterinary Physiology, Leipzig University \\ An den Tierkliniken 7, D-04103 Leipzig, Germany \\ ${ }^{2}$ Canakkale Onsekiz Mart University, Faculty of Agriculture, Department of Animal Science \\ 17100 Canakkale, Turkey
}

\begin{abstract}
This study aimed to elucidate whether sheep can increase ruminal glucose absorption acutely to counteract lactic acid formation after a concentrate meal. Pre-incubation of Ussing-chambered ruminal epithelia with D-glucose $(10 \mathrm{mM})$ on both sides for $180 \mathrm{~min}$ increased the apical uptake of $0.2 \mathrm{mM}{ }^{14} \mathrm{C}$-D-glucose. An equivalent effect was seen after serosal but not mucosal pre-incubation with D-glucose for only $10 \mathrm{~min}$. However, a 30-s serosal pre-incubation with D-glucose did not stimulate D-glucose uptake. Stimulation of uptake by serosal D-glucose was not seen after phlorizin treatment. Thus, ruminal glucose absorption via SGLT-1 can be up-regulated acutely via a basolateral glucosensor.
\end{abstract}

KEY WORDS: glucosensor, rumen, sheep, sodium glucose-linked transport, ruminal lactic acidosis

\section{INTRODUCTION}

Polysaccharides are converted by microorganisms to short chain fatty acids in the ruminant forestomach. Glucose as an intermediate metabolite does not usually reach appreciable intraruminal concentrations (Gäbel et al., 2002). When feeding larger amounts of easily fermentable carbohydrates, however, D-glucose concentrations may rise very suddenly (Ganter et al., 1993). These situations seem to be linked to a general state of dysfermentation, leading to ruminal lactic acidosis. It appears to be for those situations that ruminants retain a certain ability for D-glucose absorption by their forestomach epithelia to counteract acidosis

\footnotetext{
${ }^{*}$ Supported by the H. Wilhelm Schaumann Foundation and the Alexander von Humboldt Foundation

${ }^{3}$ Corresponding author: e-mail: aschenb@rz.uni-leipzig.de
} 
(Aschenbach et al., 2000a). The main step of D-glucose absorption is the active glucose import across the apical cell membrane via the sodium glucose-linked transporter, SGLT-1 (Aschenbach et al., 2000b). The present study was intended to elucidate whether activation of the ruminal SGLT-1 can be induced by its main substrate, D-glucose, and whether this occurs in any relevant time frame. Previously, the possibility of very rapid SGLT-1 regulation had been shown for the hormone adrenaline (Aschenbach et al., 2002).

\section{MATERIAL AND METHODS}

Isolated ruminal epithelia were prepared from adult Merino sheep at slaughter and incubated under short-circuit conditions in Ussing chambers as described by Aschenbach et al., (2000b). The oxygenated buffer solutions for washing, transport and incubation of epithelia contained (in $\mathrm{mM}$ ): $75 \mathrm{NaCl}, 5 \mathrm{KCl}, 1 \mathrm{CaCl}_{2}, 1 \mathrm{MgCl}_{2}$, $1 \mathrm{NaH}_{2} \mathrm{PO}_{4}, 2 \mathrm{Na}_{2} \mathrm{HPO}_{4}, 1 \mathrm{~L}$-glutamine, 10 sodium acetate, 10 sodium propionate, 10 butyric acid, 5 sodium D/L-lactate, 10 HEPES (free acid), 40 mannitol and 10 $\mathrm{NaOH}\left(283-293\right.$ mosmol kg-1; $\left.\mathrm{pH} 7.36-7.44 ; 37^{\circ} \mathrm{C}\right)$.

Ruminal epithelia were pre-incubated with $10 \mathrm{mM}$ D-glucose on the mucosal and/or serosal sides for periods of different length. D-Mannitol was applied equimolarly to all buffers not receiving D-glucose to compensate for any osmotic effects. The pre-incubation period with substrates was followed by a washout period without substrates. Thereafter, $0.2 \mathrm{mM}\left[{ }^{14} \mathrm{C}\right]$-labelled D-glucose was added to the mucosal solution and protein-corrected apical uptake of D-glucose was determined as described previously (Aschenbach et al., 2002). To inhibit SGLT-1, $0.1 \mathrm{mM}$ phlorizin was added to the mucosal side.

\section{RESULTS AND DISCUSSION}

To detect whether the presence of D-glucose has, at all, any influence on the activity of SGLT-1, isolated ruminal epithelia were pre-incubated on both sides with $10 \mathrm{mM}$ D-glucose for 180 and $120 \mathrm{~min}$, followed by a D-glucosefree washout period of 1 and $60 \mathrm{~min}$, respectively. These manoeuvres increased apical D-glucose uptake in comparison to control epithelia, independent of preincubation and washout time (Experiment 1 in Table 1).

A possible side specificity of the suspected substrate stimulation of SGLT1 was investigated in a second experimental series, where $10 \mathrm{mM}$ D-glucose was applied to either the mucosal or the serosal side for $10 \mathrm{~min}$, followed by a $10 \mathrm{~min}$ washout period. This shorter pre-incubation period was also effective to increase apical D-glucose uptake but only after serosal pre-incubation with $10 \mathrm{mM}$ D-glucose (Table 1). An inhibition of SGLT-1 by mucosal addition of phlorizin completely abolished the difference in D-glucose uptakes between 
control epithelia and epithelia pre-incubated with D-glucose serosally (data not shown). Consequently, the effect of serosal pre-incubation with D-glucose can be attributed specifically to an activation of SGLT-1.

The fast respo nse to serosal D-glucose in the second experimental series already suggested that the regulation does probably not involve the transcription of new messenger RNA for SGLT-1. A third experimental series was intended to elucidate whether an even shorter pre-incubation time with D-glucose would also be sufficient to stimulate SGLT-1. However, a very short pre-incubation time of 30 $\mathrm{s}$ did not produce any stimulation of SGLT-1 activity (Table 1).

The persistence of substrate stimulation was tested in a fourth experimental series by increasing the length of the washout period, i.e. the time between a 10-min substrate pre-incubation and the uptake measurement. The stimulation of D-glucose uptake by serosal pre-incubation with D-glucose disappeared when the washout period was extended to $60 \mathrm{~min}$ (Table 1). This was in contrast to the results of the first experimental series, where an increase in washout time from 1 to $60 \mathrm{~min}$ had not abolished the stimulation of apical D-glucose uptake.

Table 1. Effect of pre-incubation with $10 \mathrm{mM}$ D-glucose on D-glucose uptake

\begin{tabular}{ccccccc}
\hline \multirow{2}{*}{ Experiment } & $\mathrm{n} / \mathrm{N}$ & \multicolumn{2}{c}{ Substrate } & $\begin{array}{c}\text { Pre- } \\
\text { incubation } \\
\text { min }\end{array}$ & $\begin{array}{c}\text { Washout } \\
\text { min }\end{array}$ & $\begin{array}{c}\text { D-Glucose uptake } \\
\text { pmol mg protein }\end{array}$ \\
min $^{-1}$
\end{tabular}

M: addition of D-mannitol to exclude osmotic effects data are means and SEM of $\mathrm{n}$ observations in $\mathrm{N}$ animals statistical differences were established by ANOVA (Experiment 1) or one-way repeated measurements ANOVA (Experiment 1) 2-4), followed by Student-Newman-Keul's test values within one experimental series that do not share a common letter are different $(\mathrm{P}<0.05)$

Taken together, these data indicate that high glucose concentrations on the basolateral pole of the ruminal epithelium enhance the activity of the apical SGLT-1. 
This can be extrapolated to increased glucose absorption since apical D-glucose uptake is the rate-limiting step thereof. In contrast to the rumen, stimulation of SGLT-1 expression and/or activity of the intestine is regulated mainly by luminal D-glucose. Especially in the ovine intestine, a luminal glucosensor has been implicated in the regulation of SGLT-1 abundance after weaning when milk lactose is no longer available and other carbohydrates are largely fermented in the rumen (Wood et al., 2000). However, some up-regulation of SGLT-1 was also observed during hyperglycaemia in diabetes, which could point to additional basolateral glucosensing (Dyer et al., 2002).

The above cited previous studies attributed the increased activity of SGLT1 by luminal or systemic glucose mainly to enhanced synthesis of its mRNA transcripts. However, the time frame of stimulation in the present study does not fit transcriptional regulation of SGLT-1. Transcriptional regulation can be expected to take longer than only 10-20 min. Furthermore, with transcriptional regulation, one would have expected a fortification of the effect when increasing the pre-incubation time with D-glucose from 10 (Experiments 2-4) to 120 or 180 min (Experiment 1), which was not obvious. In contrast to a 10-min preincubation with D-glucose (Experiment 4), however, a longer pre-incubation for 120 min was able to increase the persistence of the effect (Experiment 1). This may suggest that the effect stabilizes with increasing duration of stimuli, which could be seen in line with the failure of a very short pre-incubation to stimulate SGLT-1 at all (Experiment 4).

\section{REFERENCES}

Aschenbach J.R., Bhatia S.K., Pfannkuche H., Gäbel G., 2000a. Glucose is absorbed in a sodiumdependent manner from forestomach contents of sheep. J. Nutr. 130, 2797-2801

Aschenbach J.R., Wehning H., Kurze M., Schaberg E., Nieper H., Burckhardt G., Gäbel G., 2000b. Functional and molecular biological evidence of SGLT-1 in the ruminal epithelium of sheep. Amer. J. Physiol. Gastrointest. 279, G20-G27

Aschenbach J.R., Borau T., Gäbel G., 2002. Glucose uptake via SGLT-1 is stimulated by $\beta 2-$ adrenoceptors in the ruminal epithelium of sheep. J. Nutr. 132, 1254-1257

Dyer J., Wood I.S., Palejwala A., Shirazi-Beechey S.P., 2002. Expression of monosaccharide transporters in intestine of diabetic humans. Amer. J. Physiol. Gastrointest. 282, G241-G248

Gäbel G., Aschenbach J.R., Müller F., 2002. Transfer of energy substrates across the ruminal epithelium: implications and limitations. Anim. Health Res. Rev. 3, 15-30

Ganter M., Bickhardt K., Winicker M., Schwert B., 1993. Experimental studies of the pathogenesis of rumen acidosis in sheep. Zbl. Vetmed. A 40, 731-740

Wood I.S., Dyer J., Hofmann R.R., Shirazi-Beechey S.P., 2000. Expression of the $\mathrm{Na}^{+} /$glucose co-transporter (SGLT1) in the intestine of domestic and wild ruminants. Pflügers Arch. 441, $155-162$ 